\title{
Hipertensión Arterial de pacientes con covid-19 en el Hospital General Manta.
}

\section{Arterial hypertension in patients with covid-19 at the IESS-Manta General Hospital.}

Hipertensión Arterial de pacientes con covid-19.

José Xavier López Parrales ${ }^{(1)}$

Milton Espinoza Lucas ${ }^{(2)}$

Michael Javier Castelo Caiza ${ }^{(3)}$

(1) Instituto Ecuatoriano de Seguridad social. Manta, Manabí, Ecuador. Centro Médico Particular. email: joselopez32787@ hotmail.com

https://orcid.org/0000-0002-8725-4745

(2) Universidad Laica Eloy Alfaro de Manabí. Ecuador. e-mail: milton.espinoza@uleam.edu.ec

https://orcid.org/0000-0002-8725-4745

(3) Neurólogo Independiente, e-mail:mike_nacho92@hotmail.com

https://orcid.org/0000-0002-1173-9447

\section{Resumen}

La enfermedad COVID-19 es el tercer brote epidémico de neumonía por coronavirus, causado por coronavirus SARS-CoV-2, en lo que va de este siglo. Afecta de manera más severa a grupos vulnerables, como los adultos mayores, con inmunodepresión y enfermedades crónicas entre ellas, la hipertensión arterial, de gran relevancia en la mortalidad por enfermedades cardiovasculares y cerebrovasculares, siendo un marcador clínico de gravedad de pacientes con COVID-19, manifestado fundamentalmente en edades mayores de 60 años, y la suspensión de forma preventiva de los mencionados tratamientos antihipertensivos puede conducir a complicaciones clínicas de corto a largo plazo, El objetivo del estudio fue determinar la prevalencia de la hipertensión arterial en pacientes diagnosticados con Covid-19 en el Hospital IESS-Manta, Ecuador. Se realizó un estudio de enfoque 
cuantitativo, observacional descriptivo de corte transversal, retrospectivo, en pacientes comprendidos entre los 20 hasta 65 años,que ingresaron desde Marzo hasta Octubre del 2020 al Hospital de segundo nivel y de referencia para pacientes conCOVID-19 en la Provincia de Manabí-Ecuador. Con una población de 905 sujetos diagnosticados con Covid-19 se analizaron las variables controladas de edad y sexo. El análisis estadístico se basó en la estadística descriptiva de la base de datos AS400 de la historia Clínica del Centro de Salud. La prevalencia de pacientes hipertensos diagnosticados con Covid-19 es de 58 por cada 100 pacientes que ingresaron por covid-19 y edad comprendida entre 20 a 65 equivale al $84.8 \%$.

Palabras clave: Covid-19: coronavirus SARS-CoV-2: hipertensión arterial: tratamiento antihipertensivo

\begin{abstract}
The COVID-19 disease is the third outbreak of coronavirus pneumonia, caused by the SARS-CoV-2 coronavirus, so far this century. It most severely affects vulnerable groups, such as the elderly, with immunosuppression and chronic diseases, including high blood pressure, highly relevant in mortality from cardiovascular and cerebrovascular diseases, being a clinical marker of severity of patients with COVID-19 , manifested mainly in ages older than 60 years, and the preventive suspension of the aforementioned antihypertensive treatments can lead to short- to long-term clinical complications. The objective of the study is to determine the prevalence of Arterial Hypertension in patients diagnosed with Covid- 19 at the IESS-Manta Hospital, Ecuador. A retrospective, cross-sectional, descriptive, observational, quantitative approach study was conducted in patients between 20 and 65 years old, who were admitted from March to October 2020 to the second-level Hospital and referral for patients with COVID-19 in the Province. of Manabí-Ecuador. With a population of 905 subjects diagnosed with Covid-19, the controlled variables of age and sex were analyzed. The statistical analysis was based on the descriptive statistics of the AS400 database of the Clinical History of the Health Center. The prevalence of hypertensive patients diagnosed with Covid-19 is 58 for every 100 patients who were admitted for Covid-19 and age between 20 to 65 is equivalent to $84.8 \%$.
\end{abstract}

Keywords: Covid-19: SARS-CoV-2 coronavirus: arterial hypertension: antihypertensive treatment

\title{
Introducción
}


En el año 2019, 31 de diciembre, en la Ciudad de Wuhan, localizada en Hubei, ubicada en la región Sur-central limitando al norte con Henan, al este con Anhui, al sur con Jiangxi y Hunan, al oeste con Chongqing y al noroeste con Shaanxi.Con una extensión de 187400 km, sus autoridades notificaron, que 27 personas emparentadas a un mercado de productos marinos debutaron con un síndrome respiratorio agudo de causa desconocida, siendo siete hallándose en estado grave. El 7 de enero de 2020, las autoridades chinas informaron que un nuevo coronavirus (2019-nCoV) siendo identificado como posible etiología de la enfermedad, tras realizar pruebas de estudio se descartó la presencia del SARS-CoV, el MERS-CoV, los virus de la influenza y la influenza aviar, los adenovirus, así como otras infecciones respiratorias virales o bacterianas comunes (Bernard et al., 2020; Bonilla, 2020).

Grasselli et al., (2020), aseveran que la patema afecta rápidamente a personas con sintomatología clínica, similares en otros países de Asia y, de manera creciente, a nivel mundial. La Organización Mundial de la Salud decretó, el 30 de Enero del 2020,una emergencia de salud mundial y, en febrero de 2020, se enmarcó al nuevo virus como SARS-CoV-2. El 11 de marzo de 2020 el COVID-19 desfiló a ser una pandemia (OMS, 2020).

En este sentido se ha notificado en estudios realizados en Ecuador en relación a la emergencia sanitaria del COVID-19, señalan la necesidad de estar al tanto de sus manifestaciones clínicas de riesgo, lo cual le permitirá al personal médico nivelar el pronóstico de los pacientes hospitalizados.

Al respecto es importante hacer alusión a los pacientes hipertensos afectados por la COVID-19, han tenido requerimiento de ventilación mecánica y su desenlace. Sus factores de riesgo fueron diagnóstico de hipertensión arterial. Otro aspecto relevante es las inadaptaciones, reportando práctica en el manejo de pacientes hipertensos con COVID-19, caracterizados por neumonía bilateral con manifestaciones graves de este coronavirus.

Por otra parte es importante señalar que en el primer semestre de 2020 ha permanecido bajo la influencia de la expansión sostenida de una pandemia originada por el virus SARS coronavirus 2 (SARS-CoV 2) causante de la enfermedad Covid-19, que en algunos casos provoca un desenlace fatal.

Es importante significar el trabajo publicado en la Universidad Johns Hopkins a través de Coronavirus Resource Center reporta 55292586 casos globales y una mortalidad específica para COVID-19 de 
1331895, en nuestro país Ecuador se informa de 180676 casos con 13016 decesos (Dong , Du, \& Gardner, 2020).

La evidencia actualmente disponible señala un aparente origen de selección natural de la enfermedad (Andersen, Rambaut, Lipkin, Holmes, \& Garry, 2020), lo que está afectando de manera significativa es la capacidad de transmisión y una mortalidad importante, la falta de una terapéutica curativa han coadyuvado a que estemos tratando con un serio problema de salud a nivel global (Adhikari et al.,2020).

La sintomatología que se asocia de manera rutinaria es fiebre, tos seca, mialgias, fatiga y disnea. Además, cefalea, diarrea y hemoptosis (Adhikari et al., 2020), sin embargo existen algunas variantes que se asocian a la Covid-19 que aún continúan estudiándose y van desde formas leves hasta muy graves con desenlace mortal (Chih-Cheng, Tzu-Ping, Wen-Chien, Hung-Jen, \& Po Ren, 2020).

La hipertensión arterial es una noxa que afecta a millones de persona en el mundo, y es considerada como la fuerza que ejerce la sangre circulante contra las paredes de las arterias, y se expresa en dos cifras (tensión sistólica) (tensión diastólica) (OMS, 2019).

La guía de Hipertensión arterial de la International Society of Hipertensión Global (ISH) 2020 explicita que el diagnóstico se establece cuando la presión arterial sistólica de una persona en el consultorio o clínica es igual o mayor de $140 \mathrm{mmHg}$ y/o su presión diastólica es igual o mayor de $90 \mathrm{mmHg}$ después de repetidas evaluaciones (Sociedad Colombiana de Cardiología \& Cirugía Cardiovascular, 2020).

En base a la casuística de la Organización Mundial de la Salud para el año 2019 se estima que en el mundo existen 1130 millones de personas con hipertensión, gran parte de ellos vive en países de ingresos bajos y medianos (OMS, 2019). La elevada prevalencia de la Hipertensión Arterial a nivel global y la confluencia con una enfermedad pandémica como la covid-19 plantean una asociación morbosa, que ha venido provocando cada vez mayor confluencia en la potenciación del riesgo como factores pronósticos para la hospitalización y desenlaces graves y fatales.

Las revisiones sistemáticas y los metaanálisis respaldan el criterio de asociación de comorbilidad de la hipertensión arterial y la covid-19, en 11 estudios donde se empleó el modelo de efectos fijos para estimar el efecto global, se concluyó que existe un riesgo incrementado del efecto de 4,05 (IC 95\%: 3,4-4,74; $\mathrm{p}<0,001)$ para presentar cuadros clínicos graves en pacientes con hipertensión arteria 
Diversos autores señalan que el virus afecta de una forma más grave a personas en edades avanzadas de la vida, pacientes con inmunodepresión o con enfermedades crónicas, como diabetes mellitus, cardiopatía isquémica, cáncer, enfermedad pulmonar crónica e hipertensión arterial (HTA) (Andersen, Rambaut, Lipkin, Holmes, \& Garry 2020 ; Sociedad Colombiana de Cardiología \& Cirugía Cardiovascular 2020 ).

En el continente americano se registraban hasta esa fecha un millón 134 mil 686 casos confirmados, 39,08\% del total de casos en el mundo, con 63 mil 649 fallecidos, para una letalidad de 5,62\%(-0,04). En el Ecuador hasta la fecha actual se registra 180.676 contagiados por COVID-19, casos por 1 millón de personas 10.349,94, curados 160.639, fallecidos 13.0163 (CEPAL, 2020).

El presente artículo tiene por objetivo determinar la prevalencia de la hipertensión arterial en pacientes diagnosticados con Covid-19 en el Hospital IESS-Manta, Ecuador.

\section{Predictores de mala evolución y mortalidad}

El Journal of the American Medical Association (JAMA), informa del fallecimiento de 1.625 pacientes en Italia por COVID-19 (Grasselli et al., 2020). El trabajo hace alusión a una mayor mortalidad en pacientes de edades avanzadas; los autores indican que solamente hubo 14 muertes por debajo de los 50 años; alrededor del 95\% de las muertes se observaron en mayores de 60 años, y la tasa de mortalidad se acrecentóintensamente por encima de esta edad: 3,5, 12,8 y 20,2\% para las décadas de 60-69, 70-79 y $\geq 80$ años, respectivamente.

Los datos de Italia confirmaron la asociación de la mortalidad con enfermedades cardiovasculares y diabetes; el 30\% tenían enfermedad coronaria, el 24,5\% fibrilación auricular y el 9,6\% antecedentes de accidente cerebrovascular. Solo tres pacientes $(0,8 \%)$ no tenían enfermedad subyacente y tres de cada cuatro tenían dos o más comorbilidades. Las apreciaciones de China coinciden con estos datos: que mientras la mortalidad sin comorbilidades fue del 0,9\%, se incrementó al 10,5\% con enfermedad cardiovascular, al 6,3\% con enfermedad pulmonar obstructiva crónica, al 6\% con hipertensión arterial y al 5,6\% con cáncer (Chow et al., 2020).

Al respecto el 6 de abril, la revista JAMA- Journal of the Americam Medical Association dio a conocer los datos de 1.591 pacientes internados en terapia intensiva en la Lombardía, Italia (CDC, 2020). Por otra parte se ha notificado que la hipertensión arterial (49\%) y las enfermedades cardiovasculares 
(21\%) fueron las comorbilidades más frecuentes, más que el cáncer (8\%) y que la enfermedad pulmonar obstructiva crónica (4\%) (Onder, Rezza, \& Brusaferro2020). El estudio estratificó la cohorte por la presencia o ausencia de hipertensión y los hipertensos tuvieron mayor mortalidad (65\% vs 40\%, $\mathrm{p}<0,001)$. Sin embargo, este dato debe ser tomado con precaución, pues el $58 \%$ de los pacientes continuaban internados al momento del análisis. Además, los hipertensos eran de mayor edad y la edad se relacionó con la mortalidad $(\mathrm{p}<0,001)$.

Otros ratos relevantes aportados por los Estados Unidos, muestran la asociación entre COVID-19 severo, edad avanzada y enfermedad cardiovascular (CDC, 2020).

En el reporte Morbidity and MortalityWeeklyReport (MMWR), con datos al 28 de marzo, el 78\% de los pacientes internados en terapia intensiva por COVID-19 tenían comorbilidades, y las más habituales eran la enfermedad cardiovascular (29\%) y la enfermedad pulmonar crónica (21\%). En contraste, la prevalencia de las mismas comorbilidades en pacientes no internados fue del 6, del 5 y del 7\%, respectivamente (Wu \& McGoogan 2019).

En este sentido el Centers for Disease Control and Prevention (CDC) reporta los resultados de los primeros 1.150 certificados de defunción por COVID-19: alrededor del 90\% de los fallecidos tenían más de 55 años, dato confrontable con la población inicial de China, donde el 94\% tenían más de 50 años, aunque en ambos casos parecerían algo más jóvenes que la población italiana (Lighter et al., 2020). Cabe señalar que en muchos de los estudios los análisis que evalúan la asociación entre factores de riesgo o enfermedad cardiovascular establecida y una peor evolución del COVID-19 no están ajustados por posibles confundidores, particularmente la edad, por lo que se requieren más estudios que presenten análisis multivariables.

Otros estudios publicados han evaluado la asociación de diabetes con una peor evolución del COVID19, encontrando que la prevalencia de esta patología entre quienes fallecen o requieren cuidados críticos debido a la infección por SARS-CoV-2 es elevada, rondando entre el 7,5 y el 39,5\%, según los distintos reportes (Guan et al., 2019; Grasselli et al., 2020).

Según Bhatraju et al., (2020), plantean que la obesidad se asocia con formas más severas de COVID19, un estudio realizado en pacientes en Nueva York, menores de 60 años con un índice de masa 
corporal entre 30 y $34 \mathrm{~kg} / \mathrm{m}^{2}$ tuvieron dos veces más que requerir cuidados intensivos por COVID-19, en comparación con pacientes con un índice de masa corporal menor a $30 \mathrm{~kg} / \mathrm{m}^{2}$.

\section{Materiales y Métodos}

El estudio es de naturaleza observacional, de corte transversal que responde a un enfoque cuantitativo, hombres y mujeres de edades de 20 años hasta 65 años, donde se analizará la variable dependiente Hipertensión Arterial, la variable independiente COVID-19 y las variables controladas de edad, sexo y otras comorbilidades. Los métodos empleados corresponden al nivel descriptivo.

La investigación se realizó en un Hospital de segundo nivel y de referencia para pacientes con diagnóstico de COVID-19 en la Provincia de Manabí-Ecuador. Siendo consensuada la autorización, para la obtención de los datos estadísticos, por parte de las autoridades del Hospital General Manta, departamento de docencia, logrando obtener acceso a la base de datos, con fines netamente investigativos.

Los datos estadísticos fueron obtenidos en base a las morbilidades de los pacientes atendidos en el Hospital General de Manta, desde el mes de Marzo hasta el mes de Octubre del año 2020 y emitidos por el área de Planificación y Estadística, extraídos del sistema MIS AS-400.

La población estuvo conformada por 905 pacientes diagnosticas con la Covid-19 recuperados de la base de datos.

El análisis estadístico se basó en la estadística descriptiva y se desarrolló con la aplicación Excel en su versión 15.

Se determinaron los siguientes criterios:

\section{Criterios de inclusión:}

a) Hombres y mujeres, edades comprendidas desde los 20 a 65 años.

b) Hipertensión Arterial más Diagnóstico de COVID-19.

\section{Criterios de exclusión y eliminación}


a) Pacientes derivados a otras casas de salud.

b) Pacientes qué solicitaron alta petición del Hospital General Manta.

c) Pacientes fallecidos por COVID-19 con diagnóstico de Hipertensión Arterial.

d) Pacientes sin datos en la Historia Clínica de referencia.

\section{Resultados}

En la gráfica 1, se observa el total de 905 pacientes con diagnóstico de COVID-19, de los cuáles 529 corresponden a pacientes con Hipertensión Arterial.

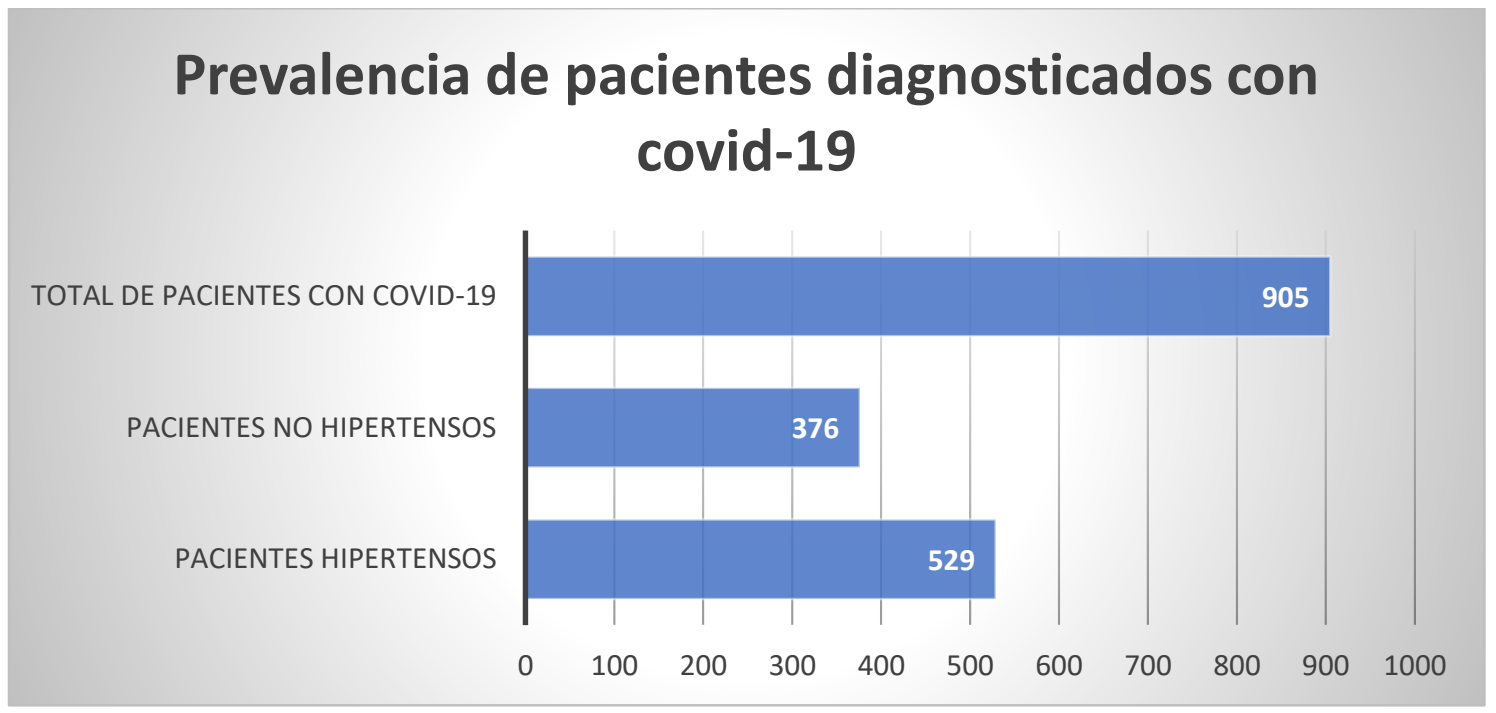

Gráfico 1. Prevalencia de pacientes diagnosticados con COVID 19.

\begin{tabular}{|l|l|l|}
\hline & Prevalencia & Porcentaje \\
\hline Hipertensos con Covid-19 & 529 & 58.4 \\
\hline No hipertensos con Covid-19 & 376 & 41.6 \\
\hline Total de pacientes con covid-19 & 905 & 100 \\
\hline
\end{tabular}

Tabla 1. Prevalencia de pacientes con covid-19 e hipertensión. Fuente: Sistema MIS AS-400

En lo relativo a la gráfica 2, se observa el total de 905 pacientes correspondientes desde de 0 a 99 años, con diagnóstico de COVID-19, seguidamente pacientes correspondientes de las edades de 20 a 65 años, los 529 pacientes corresponden a los pacientes hipertensos. 


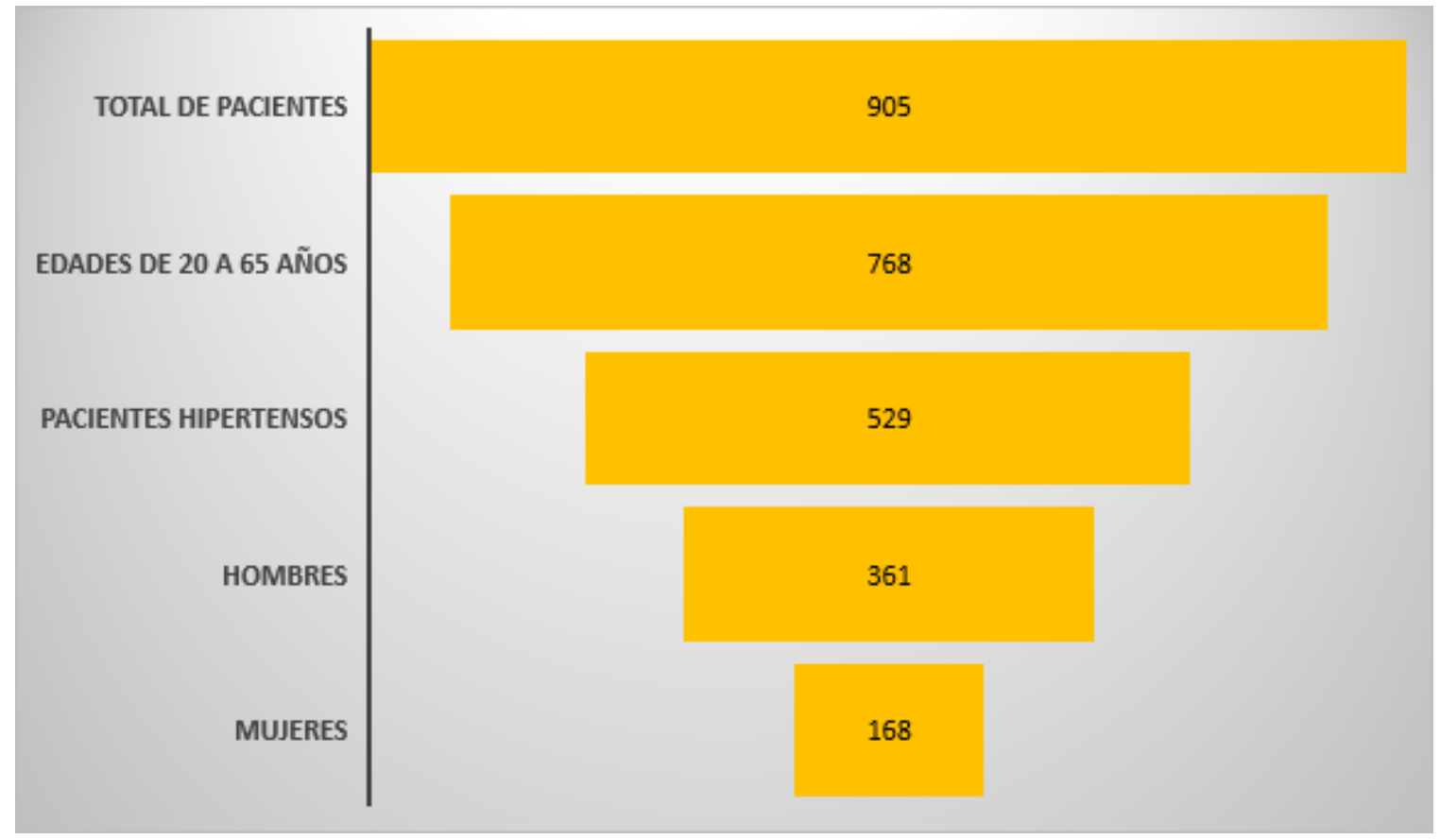

Gráfica 2. Pacientes por edades afectados de COVID-19.

\section{Discusión}

En cuanto a los pacientes con diagnóstico de hipertensión arterial más la afectación por COVID-19 descritos en el actual estudio corresponde a los atendidos en el Hospital General Manta comprendido desde los meses de Marzo hasta Octubre. En este estudio se concuerda con otras investigaciones realizadas China y en los Estados Unidos de Norteamérica, muestran una forma de enfermedad preponderante en varones que inicia con tos y otros síntomas respiratorios, con un tiempo de enfermedad promedio de siete días (Bhatraju et al., 2020; Yang et al., 2020). Otros autores hacen alusión a la fiebre y la disnea, plantean que son síntomas menos frecuentes y no sería adecuado tomarlos como criterio diagnóstico o de gravedad (Zhang et al., 2020).

Se concuerda con Shi, Yu, Huang, \& Tan (2019), en la que hacen referencia a considerar datos objetivos, como saturación de oxígeno capilar o escores clínicos de fácil acceso para la atención inicial, y tomar acciones en el paciente. Otros investigadores indican que los síntomas gastrointestinales son presentaciones atípicas y poco frecuentes (Yoon et al. 2019).

Los historias de exámenes complementarios en COVID-19 son escasos, de nuestro estudio por datos obtenidos a través del Hospital General Manta por medio del sistema MIS AS-400,prevaleceelevación 
de reactantes de fase aguda como proteína $\mathrm{C}$ reactiva y linfopenia, análogo a lo instruido en China o en los EE. UU. Respecto al punto de corte para considerar linfopenia, esta fue menor de 900 linfocitos por milímimetro cúbico en comparación a los 1500 por $\mathrm{mm}^{3}$ que se consideró en los EE. UU. Llama la atención la mayor frecuencia de elevación de enzimas hepáticas como las transaminasas frente a lo observado en otros países (Yoon et al., 2020; Zhang et al., 2020).

Se concuerda con otros estudios que hacen referencia una mayor afectación en pacientes del sexo masculino comprendido desde la edad de 20 a 65 años, con total de 361 diagnosticados de hipertensión arterial, seguido del sexo femenino con un total de 168 pacientes con iguales características clínicas (Zhang et al., 2020).

Otros estudios sobre las imágenes tomográficas corresponden principalmente torácicas sin contraste, dónde se evidencian algunos casos cardiomegalia pacientes de larga dato de evolución de Hipertensión Arterial, lesiones en vidrio esmerilado, similares a lo sujeto en China y Corea (Yoon et al.2019).

A manera de reflexión podemos plantear que no existe hasta el momento, tratamiento comprobado para COVID-19, en el presente estudio se asocian antibióticos de amplio espectro, similares a los utilizados en otros países (Bhatraju et al.2020). Desde un inicio se utilizó azitromicina, principalmente por ser parte del tratamiento para neumonía atípica, en cambio la hidroxicloroquina se pudo emplear semanas después, cuando se tuvo autorización hospitalaria para su uso. El apoyo ventilatorio invasivo fue relativamente menor, pero es una serie pequeña de casos y al inicio se demoró su inicio, sumado a la medicación habitual de la Hipertensión Arterial.

De esta manera, los problemas de organización durante inicio de esta pandemia provocada por COVID19, los días de atención en el Hospital General Manta, evaluado pudieron haber influido en la evolución de los pacientes, debido a la falta de exámenes hematológicos, bioquímicos por inconvenientes con la bioseguridad personal y ambiental. Anteponiéndose limitaciones principalmente, datos clínicos y resultados de exámenes complementarios incompletos y criterios diagnósticos no lineales. No se investigó infección concomitante con otros virus ni complicaciones bacterianas.

\section{Conclusiones}

La investigación realizada refleja una de las primeras experiencias, en un hospital ecuatoriano, el Hospital General Manta, siendo parte de la Red Integral Pública de Salud, hospital centinela, brindando 
atención con COVID-19, caracterizados por neumonía bilateral, más frecuente en varones, con una comorbilidad importante la Hipertensión Arterial por encima del $50 \%$, y con una frecuencia centralizada en el rango de edad de 20 a 65 años.

Se confirma que el estudio revela la importancia y la urgencia de ser capaces de reconocer el cuadro clínico de esta enfermedad y así conocer su mecanismo de transmisión, evitando la propagación de este virus, además de vincular la comorbilidades de enfermedades crónicas no transmisibles como efecto deletéreo en la Salud global.

\section{Bibliografía}

Adhikari, S., Meng, S., Wu, Y., Mao, Y., Ye, R., Wang , Q., et al. (2020). Epidemiology, causes, clinical manifestation and diagnosis, prevention and control of coronavirus disease (COVID-19) during the early outbreak period: a scoping review. Infectious Diseases of Poverty, 29.

Andersen, K., Rambaut, A., Lipkin, W., Holmes, E., \& Garry, R. (2020). The proximal origin of SARS-CoV 2. Nature Medicine, 450-455.Recuperado de: https://www.nature.com/articles/s41591-020-0820-9.pdf

Bernard, S., Rolland, P., Silue, Y., Mailles, A., Campese,. C, Simondon. A., et al. (2019) First cases of coronavirus disease 2019 (COVID-19) in France: surveillance, investigations and control measures, January 2020. Euro Surveill, 25 (6). doi: 10.2807/15607917.ES.2020.25.6.2000094.

Bonilla, O. (2020). Para entender la COVID-19. Revista Medicentro Electrónica, 24(3), 595-629. Recuperado de: http://scielo.sld.cu/pdf/mdc/v24n3/1029-3043-mdc-24-03-595.pdf

Bhatraju, P., Ghassemieh, B., Nichols, M., Kim, R., Jerome, K., Nalla, A., et al. (2020). Covid-19 in Critically Ill Patients in the Seattle Region - Case Series. N Engl J Med. doi: 10.1056/NEJMoa2004500.

CDC. National Health Statistics.(2020). Recuperado de: https://www.cdc.gov/media/dpk/diseases-andconditions/coronavirus/coronavirus-2020.html

CEPAL. (2020). La pandemia del COVID-19 profundiza la crisis de los cuidados en América Latina y el Caribe. Recuperado de: https://repositorio.cepal.org/bitstream/handle/1136211362/45335/S2000261_es.pdf?sequence=5 \&isAllowed $=\mathrm{y}$ 
Chih- Cheng, L., Tzu-Ping, S., Wen-Chien, K., Hung Jen, T., \& Po Ren, H. (2020). Severe acute respiratory syndrome coronavirus 2 (SARS-CoV-2) and corona virus disease-2019 (COVID-19): the epidemic and the challenges. Recuperado el 16 de 11 de 2020, de International Journal of Antimicrobial Agents.doi.org/10.1016/j.ijantimicag. 2020.105924

Chow, K. Fleming-Dutra, R. Gierke, A. Hall, M.M. Hughes, T. Pilishvili, et al. $\quad$ (2020). Preliminary estimates of the prevalence of selected underlying health conditions among patients with coronavirus disease 2019 -United States. Morb Mortal Wkly Rep., 69. 382-386. doi: 10.15585/mmwr.mm6913e2

Dong, E., Du, H., \& Gardner, L. (2020). An interactive web-based dashboard to track COVID - n real time. Lancet Inf Dis., 533-534.

Guan, W., Ni, Z., Hu., Y., Liang, WH., Ou. CQ., He, JX., et al. (2019).Clinical Characteristics of Coronavirus Disease 2019 in China. N Engl J Med. (28)1-13. doi: 10.1056/NEJMoa2002032.

Grasselli, A. Zangrillo, A. Zanella, M. Antonelli, L. Cabrini, A. Castelli, et al. (2020). Baseline characteristics and outcomes of 1591 patients infected with SARS-CoV-2 admitted to ICUs of the Lombardy Region, Italy JAMA. doi: 10.1001/jama.2020.5394

Lighter, M., Phillips, S., Hochman. S., Sterling, D., Johnson, F. Francois, et al. ～(2020), Obesity Obesity in patients younger than 60 years is a risk factor for COVID-19 hospital admission Clin. Infect. Dis.

Onder, G. Rezza, G. Brusaferro, S. (2020). Case-fatality rate and characteristics of patients dying in relation to COVID-19 in ItalyJAMA. doi: 10.1001/jama.2020.4683

Organización Mundial de la Salud. (2019). Hipertensión. Recuperado de: https://www.who.int/es/news-room/fact-sheets/detail/hypertension

Organización Mundial de la Salud. (2020).Coronavirus disease 2019 (COVID-19). Situation Report66.Geneva: WHO. Recuperado de: https://www.who.int/docs/defaultsorce/ coronaviruse/situation-reports/20200326-sitrep-66-covid-19.pdf?sfvrsn=9e5b8b48_2

Sociedad Colombiana de Cardiología \& Cirugía Cardiovascular. (2020). Asociación de la Sociedad Colombiana de Cardiología \& Cirugía Cardiovascular. Recuperado de: https://scc.org.co/boletinno-144-principales-mensajes-de-las-guias-de-hipertension-arterial-de-la-ish-en-el-2020/

Shi F, Yu Q, Huang W, Tan C. (2020). Novel Coronavirus (COVID-19) Pneumonia with Hemoptysis as the Initial Symptom: CT and Clinical Features. Korean J Radiol. 21:e42. doi://10.3348/kjr.2020.0181. 
Wang, L.S., Wang, Y.R., Ye, D.W., Liu, Q. (2020).A review of the 2019 novel coronavirus (SARS$\mathrm{CoV}-2$ ) based on current evidence. Int J AntimicrobAgents. Recuperado de: https://www.ncbi.nlm.nih.gov/pmc/articles/PMC7156162/

Wu, Z., \& McGoogan. J.M.(2019). Characteristics of and important lessons from the coronavirus disease 2019 (COVID-19) outbreak in China JAMA., 323,1239-1242. doi: 10.1001/jama.2020.2648

Yang X, Yu Y, Xu J, Shu H, Xia J, Liu H, et al. Clinical course and outcomes of critically ill patients with SARS-CoV-2 pneumonia in Wuhan, China: a single-centered, retrospective, observational study. Lancet Respir Med. 2020;(20):1-7 [Epub ahead of print]. doi: 10.1016/S22132600(20)30079-5.

Yoon SH, Lee KH, Kim JY, Lee YK, Ko H, Kim KH, et al. (2020).Chest Radiographic and CT Findings of the 2019 Novel Coronavirus Disease (COVID-19): Analysis of Nine Patients Treated in Korea. Korean J Radiol. 21(4):494-500. doi: 10.3348/kjr.2020.0132.

Zhang J, Dong X, Cao Y, Yuan Y, Yang Y, Yan Y, et al. Clinical characteristics of 140 patients infected with SARS-CoV-2 in Wuhan, China. Allergy Eur J Allergy Clin Immunol. 1-12. doi: 10.1111/all.14238. 